\title{
Time-critical neurological emergencies: the unfulfilled role for point-of-care testing
}

\author{
Jason T. McMullan • William A. Knight • \\ Joseph F. Clark • Fred R. Beyette • Arthur Pancioli
}

Received: 4 December 2009 /Accepted: 5 March 2010/Published online: 18 May 2010

(C) Springer-Verlag London Ltd 2010

\begin{abstract}
Background Neurological emergencies are common and frequently devastating. Every year, millions of Americans suffer an acute stroke, severe traumatic brain injury, subarachnoid hemorrhage, status epilepticus, or spinal cord injury severe enough to require medical intervention.

Aims Full evaluation of the diseases in the acute setting often requires advanced diagnostics, and treatment frequently necessitates transfer to specialized centers. Delays in diagnosis and/or treatment may result in worsened outcomes; therefore, optimization of diagnostics is critical.

Methods Point-of-care (POC) testing brings advanced diagnostics to the patient's bedside in an effort to assist medical providers with real-time decisions based on real-time infor-
\end{abstract}

The views expressed in this paper are those of the author(s) and not those of the editors, editorial board or publisher.

J. T. McMullan $(\bowtie) \cdot$ W. A. Knight $\cdot$ A. Pancioli

Department of Emergency Medicine,

University of Cincinnati,

231 Albert Sabin Way,

PO Box 670769,

Cincinnati, OH 45267-0769, USA

e-mail: Jason.McMullan@uc.edu

\section{W. A. Knight}

Department of Neurosurgery,

University of Cincinnati,

Cincinnati, OH, USA

J. F. Clark

Department of Neurology,

University of Cincinnati,

Cincinnati, OH, USA

\section{F. R. Beyette}

Department of Electrical Engineering,

University of Cincinnati,

Cincinnati, OH, USA mation. POC testing is usually associated with blood tests (blood glucose, troponin, etc.), but can involve imaging, medical devices, or adapting existing technologies for use outside of the hospital. Noticeably missing from the list of current point-of-care technologies are real-time bedside capabilities that address neurological emergencies.

Results Unfortunately, the lack of these technologies may result in delayed identification of patients of these devastating conditions and contribute to less aggressive therapies than is seen with other disease processes. Development of timedependent technologies appropriate for use with the neurologically ill patient are needed to improve therapies and outcomes. Conclusion POC-CENT is designed to support the development of novel ideas focused on improving diagnostic or prognostic capabilities for acute neurological emergencies. Eligible examples include biomarkers of traumatic brain injury, non-invasive measurements of intracranial pressure or cerebral vasospasm, and improved detection of pathological bacteria in suspected meningitis.

Keywords Point-of-care systems · Neurology · Diagnostic techniques, neurological - Emergency medicine $\cdot$ Brain . Critical care

Neurological emergencies are common and frequently devastating. Every year, millions of Americans suffer an acute stroke, severe traumatic brain injury, subarachnoid hemorrhage, status epilepticus, or spinal cord injury severe enough to require medical intervention [1-10]. Full evaluation of the diseases in the acute setting often requires advanced diagnostics, and treatment frequently necessitates transfer to specialized centers. Delays in diagnosis and/or treatment may result in worsened outcomes therefore optimization of diagnostics is critical. 


\section{Acute ischemic stroke}

Worldwide, acute ischemic stroke affects millions of people every year, causing significant morbidity and mortality; in the US, a person suffers a stroke every $40 \mathrm{~s}$, and a stroke death occurs every $4 \mathrm{~min}$ [2]. The annual age-adjusted incidence per 100,000 population is 105-262 in India [11] and 140.1/ 100,000 in Chile [12], compared to $158 / 100,000$ in the US [2]. The use of recombinant rt-PA is strictly limited by time, and a large number of patients present outside the window for treatment. Even for those who are treated, functional outcome and the time interval between stroke symptom onset and drug administration are inversely related (Fig. 1) $[13,14]$.

Treatment decisions require non-contrast computed tomography (CT) to rule out intracranial hemorrhage; other stroke mimics are not uncommon [15]. While CT technology is readily available in most cities, rural settings and developing countries may not have rapid guaranteed access to this diagnostic tool or the skilled personnel to interpret the images. Even when CT is available, time is dedicated to the acquisition and interpretation of the images before treatment can begin, causing an unavoidable and highly variable delay in therapy.

Alternative techniques that can assist in real-time diagnostic separation of ischemic from hemorrhagic strokes, with minimal subjective interpretation, are needed. Such novel methods could speed time to therapy or increase the number of stroke patients who would be eligible for treatment, thereby improving outcomes. This technology could serve to differentiate likely candidates from unlikely candidates and aid in prioritization of CT scans. In addition, diagnostics could be translated into the pre-hospital setting, allowing Emergency Medical Services to triage patients to the most appropriate facilities - subjects with intracranial

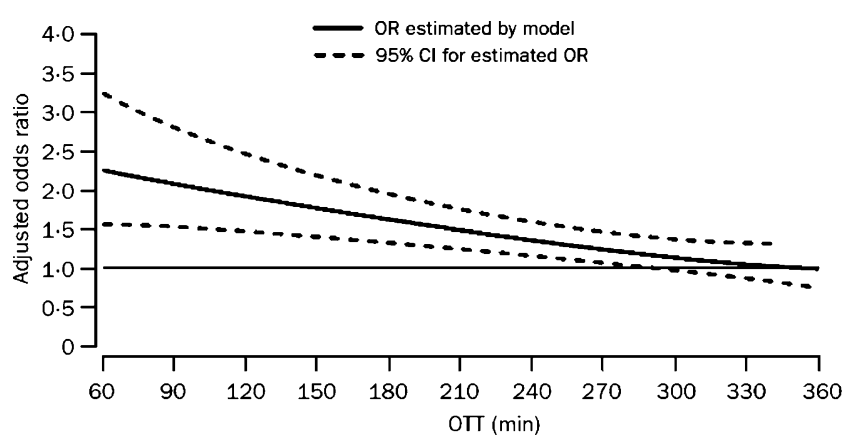

Fig. 1 Odds ratio of favorable outcome after acute ischemic stroke is dependent on symptom onset to rT-pa treatment interval. (Borrowed with permission from Hacke W, Donnan G, Fieschi C, Kaste M, von Kummer R, Broderick JP, et al. Association of outcome with early stroke treatment: pooled analysis of ATLANTIS, ECASS, and NINDS rt-PA stroke trials. Lancet 2004;363(9411):768-74) hemorrhage could be taken to neurosurgical capable facilities, while those with ischemic stroke could be taken to the closest appropriate facility for rt-PA administration. Uncoupling the reliance on $\mathrm{CT}$ to determine treatment could, one day, allow outside-of-hospital therapy options as many stroke victims first present to primary care physician offices or face prolonged ambulance transport times [16]. Pre-hospital systems that utilize physicians could, one day, administer rt-PA prior to hospital arrival if hemorrhagic stroke could be excluded, or ischemic infarction definitively diagnosed, with point-of-care technologies.

The fear of causing intracranial hemorrhage is often cited as a reason why thrombolytic therapy is not offered by treating physicians [17, 18]. Advanced age, duration of symptoms, and severity of stroke increase the risk of hemorrhage. Hemorrhagic transformation of ischemic stroke with the use of rt-PA remains unpredictable in individual scenarios [19], and treatment response and clinical improvement are variable. The result is that clinicians can only offer an educated guess of the chance of recovery or worsening for individual patients based on treatment choices. A real-time, point-of-care test that will better predict risk of hemorrhage or probability of good response to therapy with the expected benefit of decreasing fear and risk of intracranial hemorrhage is needed for these clinical scenarios. These tests could help health care providers, patients, and families weigh true riskbenefit ratios when deciding a treatment course.

\section{Non-convulsive status epilepticus}

Non-convulsive status epilepticus (NSCE) is a challenging clinical diagnosis with potentially devastating consequences if untreated [20]. Like convulsive status epilepticus, nonconvulsive seizures are more likely to be controlled when treated early, and the length of seizure activity is correlated with poor outcomes [21-23]. NCSE may be present in patients with unexplained coma or altered mental status; some patients may be able to follow commands despite epileptic activity on EEG [24]. This is of special concern in critically ill patients and may complicate the course of up to half of those in a coma $[24,25]$. NCSE is prevalent in patients with traumatic brain injury, subarachnoid hemorrhage, hemorrhagic and ischemic stroke, and cerebral malignancy, where these seizures may contribute to secondary injuries and worsened outcomes [26]. NCSE may mimic acute ischemic stroke [27], and such patients are occasionally treated with rt-PA. Differentiating NCSE from acute stroke would minimize such treatments and decrease concomitant risks associated with rt-PA administration.

Currently, diagnosis of non-convulsive status requires continuous EEG monitoring, which is generally only 
available at tertiary referral centers. Even when available, the time required to place electrodes and the resources needed to provide skilled monitoring and interpretation make continuous EEG monitoring onerous in most centers. Because treatment and recovery are time-dependent and diagnosis is difficult, alternatives to traditional EEG monitoring are needed. Point-of-care diagnostics with easy to interpret and real-time results could improve the care of many patients.

\section{Suspected traumatic brain injury}

Altered mental status after trauma is common, but does not always represent a traumatic brain injury (TBI). In some cases, pre-existing medical conditions, antecedent medical emergencies, or intoxicants cloud the clinical picture. Mechanism of injury is not a reliable predictor of underlying injuries, and Emergency Medical Services frequently overtriage these patients to designated Trauma Centers [28]. Helicopter transport from a trauma scene is often requested due to suspicion of possible TBI for patients who ultimately do not have significant injuries [28]. Emergency Departments often obtain head CTs that ultimately prove to be normal, and existing clinical decision rules have not diminished this practice because of broad inclusion criteria and a reluctance to miss high morbidity injuries [29, 30]. Further, normal head CTs do not guarantee that severe injury is not present; initial radiographic findings in diffuse axonal and global hypoxic injuries may be unremarkable even in a clinically devastated patient. These scenarios represent a gap in the ability to detect neuropathological events in the TBI patient. Improved diagnostic modalities may aid in the triage, transport, and treatment of victims of acute neurological emergencies.

Delayed recognition and treatment of severe TBI may be harmful. Aggressive airway, ventilatory, and intracranial pressure management in concert with efficient neurosurgical decompression and hematoma evacuation may be life saving. Conversely, endotracheal intubation or use of osmotic agents for intracranial pressure control in patients without TBI poses possible harm without potential for benefit. Improvements in the diagnostic capabilities in this patient population are needed to better triage resources and separate those most at-risk from those not at risk.

\section{Meningitis}

The diagnosis of meningitis is, in itself, relatively straightforward and relies on analysis of cell counts in cerebrospinal fluid samples. Improved technology could speed the time needed to analyze these samples, but clinical impact would likely be minimal. Differentiation of meningitis etiology - bacterial, viral, fungal, or sterile - without awaiting culture results would have a much larger impact on clinical care [31]. Anti-infectious agents could be tailored in an expedient manner, steroids could be given only to those most likely to benefit, and outpatient management strategies could be developed for the care of patients at lowrisk of poor outcomes [32].

Current practice is to treat with empiric broad-spectrum antibiotics and anti-virals for $24-48 \mathrm{~h}$ while awaiting CSF culture results; this therapy is only available as an inpatient, requiring hospital admission for many patients who ultimately have non-bacterial, non-HSV meningitis that require symptomatic support [33]. In developed countries, approximately 4 per 100,000 persons per year develop bacterial meningitis, resulting in sizable outlays of monetary and clinical resources [32]. Development of a point-ofcare technology that could quickly differentiate bacterial from non-bacterial meningitis, for example, could curtail the current necessary over-triage and over-treatment of patients with suspected meningitis.

\section{Point-of-care testing}

Point-of-care (POC) testing brings advanced diagnostics to the patient's bedside in an effort to assist medical providers with real-time decisions based on real-time information. POC testing is usually associated with blood tests (blood glucose, troponin, etc.), but can involve imaging, medical devices, or adapting existing technologies for use outside of the hospital. Successful POC testing should impact aspects of the triage, transport, and/or treatment of the patient.

The care of patients with acute myocardial infarction has been accelerated by the use of pre-hospital EKGs, greatly reducing medical contact to balloon times for percutaneous coronary interventions; paramedic administered fibrinolytics have been attempted for decades based on integration of this POC diagnostic tool [34, 35]. Ultra-sensitive bedside troponin assays decrease the chance of missed myocardial infarctions and speed time to therapy in non-ST segment elevation myocardial infarction patients [36]. Such rapid testing also allows early identification of subjects for clinical trials [37].

The care of the septic patient has been revolutionized by the development of real-time markers of systemic hypoperfusion, particularly lactate and central venous oxygen saturation $\left(\mathrm{SCVO}_{2}\right)$. The concept of an early goal directed therapy in sepsis has decreased mortality, in part due to improved recognition and identification of patients [38]. Lactate clearance and improvement in $\mathrm{SCVO}_{2}$ are objective measures of response to therapy and assist treating 
physicians in therapeutic decisions. Technology has advanced to allow continuous measurement of these markers at the bedside through advanced central catheters [39].

Management of the severely traumatized patient has improved with the use of bedside ultrasonography. Pneumothorax, pericardial effusion, and hemoperitoneum can be quickly identified and used to help guide therapy. The development of smaller and sturdier devices has created a niche for the out-of-hospital environment, with obvious implications for EMS, combat casualty care, and disaster and mass casualty situations [40].

Noticeably missing from the list of current point-of-care technologies are real-time bedside capabilities that address neurological emergencies. The most common POC test used for altered mental status patients is blood glucose level, which allows rapid diagnosis and treatment of hypoglycemia or exclusion of this process from a differential diagnosis. Continuous EEG monitoring, as discussed above, can be considered a POC technology. The complexity of EEG interpretation and time and precision required for proper electrode placement limit the utility of EEG outside of controlled and specialized settings. Ultrasonic measurement of optic nerve sheath diameter is being explored as a POC test for intracranial hypertension; this field of study is expanding, but not yet ready to be applied in routine clinical practice [4143]. Unfortunately, the lack of more of these types of technologies may result in delayed identification of patients with these devastating conditions and contribute to less aggressive therapies than are seen with other disease processes. Development of time-dependent technologies appropriate for use with the neurologically ill patient is needed to improve therapies and outcomes.

\section{Current funding opportunities}

Development of novel techniques and technologies requires significant funding, which can be difficult to obtain without preliminary data. Many investigators rely on intra-mural funding and donated academic effort to conduct proof-ofconcept pilot studies. While this "bootstrap" approach to new product initiation can be personally rewarding, such professional devotion can be an entry barrier for new investigators. In partial response to this need, the National Institutes of Health (NIH) have funded the Point-of-Care Technologies Research Network (POCTRN) [44]. Each center in the Network is funded to support the development of point-of-care technologies in a specific clinical area. The Point-of-Care Center for Emerging Neurologic Technologies (POC-CENT) at the University of Cincinnati (funded by grant no. 5-U54-EB007954) is focused towards generating diagnostics appropriate for acute neurological emergencies where point-of-care information can be obtained.
Like all of the Centers in POCTRN, POC-CENT uses clinical-needs driven decision making to target both early stage technological development and pre-clinical assessment of emerging point-of-care technologies. In addition to supporting projects that are internal to the Center, POCCENT can fund external research and/or work as a collaborator with research scientists and clinicians to develop new technology concepts. Any entity that is eligible for NIH funding including faculty, post docs, residents, and corporate industry can be supported by POC-CENT.

POC-CENT is designed to support the development of novel ideas focused on improving diagnostic or prognostic capabilities for acute neurological emergencies. Eligible examples include biomarkers of traumatic brain injury, noninvasive measurements of intracranial pressure or cerebral vasospasm, and improved detection of pathological bacteria in suspected meningitis.

\section{Conclusion}

Acute neurological emergencies pose a significant threat to the life and independence of every person. Often, such emergencies strike without warning, and medical care must be provided as soon as possible to minimize significant and permanent functional impairments. Currently, unavoidable delays required to obtain confirmatory testing impact the speed at which this time-critical care can be delivered. POC technologies that impact the triage, transport, or treatment of these patients are critically needed. As creative scientists and clinicians imagine ways to improve diagnostic techniques, POC-CENT exists to support and enable the transition from invention to intervention.

\section{References}

1. Consensus conference (1999) Rehabilitation of persons with traumatic brain injury. NIH Consensus Development Panel on Rehabilitation of Persons With Traumatic Brain Injury. JAMA 282(10):974-983

2. Rosamond W, Flegal K, Furie K, Go A, Greenlund K, Haase N et al (2008) Heart disease and stroke statistics-2008 update: a report from the American Heart Association Statistics Committee and Stroke Statistics Subcommittee. Circulation 117(4):e25-e146

3. Bassin S, Smith TL, Bleck TP (2002) Clinical review: status epilepticus. Crit Care 6(2):137-142

4. Broderick JP, Brott T, Tomsick T, Miller R, Huster G (1993) Intracerebral hemorrhage more than twice as common as subarachnoid hemorrhage. J Neurosurg 78(2):188-191

5. Brown AW, Leibson CL, Malec JF, Perkins PK, Diehl NN, Larson DR (2004) Long-term survival after traumatic brain injury: a population-based analysis. NeuroRehabilitation 19(1):37-43 
6. Qureshi AI, Tuhrim S, Broderick JP, Batjer HH, Hondo H, Hanley DF (2001) Spontaneous intracerebral hemorrhage. N Engl J Med 344(19):1450-1460

7. Schievink WI (1997) Intracranial aneurysms. N Engl J Med 336 (1):28-40

8. Sekhon LH, Fehlings MG (2001) Epidemiology, demographics, and pathophysiology of acute spinal cord injury. Spine (Phila Pa 1976) 26(24 Suppl):S2-S12

9. Williams GR, Jiang JG, Matchar DB, Samsa GP (1999) Incidence and occurrence of total (first-ever and recurrent) stroke. Stroke 30 (12):2523-2528

10. Wu YW, Shek DW, Garcia PA, Zhao S, Johnston SC (2002) Incidence and mortality of generalized convulsive status epilepticus in California. Neurology 58(7):1070-1076

11. Banerjee TK, Das SK (2006) Epidemiology of stroke in India. Neurol Asia 11:1-4

12. Lavados PM, Sacks C, Prina L, Escobar A, Tossi C, Araya F et al (2005) Incidence, 30-day case-fatality rate, and prognosis of stroke in Iquique, Chile: a 2-year community-based prospective study (PISCIS project). Lancet 365(9478):2206-2215

13. The National Institute of Neurological Disorders and Stroke rt-PA Stroke Study Group (1995) Tissue plasminogen activator for acute ischemic stroke. N Engl J Med 333(24):1581-1587

14. Hacke W, Donnan G, Fieschi C, Kaste M, von Kummer R, Broderick JP et al (2004) Association of outcome with early stroke treatment: pooled analysis of ATLANTIS, ECASS, and NINDS rt-PA stroke trials. Lancet 363(9411):768-774

15. Scott PA, Silbergleit R (2003) Misdiagnosis of stroke in tissue plasminogen activator-treated patients: characteristics and outcomes. Ann Emerg Med 42(5):611-618

16. Kothari R, Jauch E, Broderick J, Brott T, Sauerbeck L, Khoury J et al (1999) Acute stroke: delays to presentation and emergency department evaluation. Ann Emerg Med 33(1):3-8

17. Brown DL, Barsan WG, Lisabeth LD, Gallery ME, Morgenstern LB (2005) Survey of emergency physicians about recombinant tissue plasminogen activator for acute ischemic stroke. Ann Emerg Med 46(1):56-60

18. Weintraub MI (2006) Thrombolysis (tissue plasminogen activator) in stroke: a medicolegal quagmire. Stroke 37(7):1917-1922

19. The NINDS t-PA Stroke Study Group (1997) Intracerebral hemorrhage after intravenous t-PA therapy for ischemic stroke. Stroke 28(11):2109-2118

20. Shah AM, Vashi A, Jagoda A (2009) Review article: Convulsive and non-convulsive status epilepticus: an emergency medicine perspective. Emerg Med Australas 21(5):352-366

21. Haut SR, Veliskova J, Moshe SL (2004) Susceptibility of immature and adult brains to seizure effects. Lancet Neurol 3 (10):608-617

22. Maegaki Y, Kurozawa Y, Hanaki K, Ohno K (2005) Risk factors for fatality and neurological sequelae after status epilepticus in children. Neuropediatrics 36(3):186-192

23. Morimoto K, Fahnestock M, Racine RJ (2004) Kindling and status epilepticus models of epilepsy: rewiring the brain. Prog Neurobiol 73(1):1-60

24. Meierkord H, Holtkamp M (2007) Non-convulsive status epilepticus in adults: clinical forms and treatment. Lancet Neurol 6 (4):329-339

25. Claassen J, Mayer SA, Kowalski RG, Emerson RG, Hirsch LJ (2004) Detection of electrographic seizures with continuous EEG monitoring in critically ill patients. Neurology 62(10):1743-1748

26. Vespa PM, Nuwer MR, Nenov V, Ronne-Engstrom E, Hovda DA, Bergsneider $M$ et al (1999) Increased incidence and impact of nonconvulsive and convulsive seizures after traumatic brain injury as detected by continuous electroencephalographic monitoring. J Neurosurg 91(5):750-760

27. Behrens JM (1980) Psychomotor status epilepticus masking as a stroke. Postgrad Med 68(5):223-4, 226

28. Ringburg AN, de Ronde G, Thomas SH, van Lieshout EM, Patka P, Schipper IB (2009) Validity of helicopter emergency medical services dispatch criteria for traumatic injuries: a systematic review. Prehosp Emerg Care 13(1):28-36

29. Haydel MJ, Preston CA, Mills TJ, Luber S, Blaudeau E, DeBlieux PM (2000) Indications for computed tomography in patients with minor head injury. N Engl J Med 343(2):100-105

30. Stiell IG, Wells GA, Vandemheen K, Clement C, Lesiuk H, Laupacis A et al (2001) The Canadian CT Head Rule for patients with minor head injury. Lancet 357(9266):1391-1396

31. Dubos F, Martinot A, Gendrel D, Breart G, Chalumeau M (2009) Clinical decision rules for evaluating meningitis in children. Curr Opin Neurol 22(3):288-293

32. Fitch MT, van de Beek D (2007) Emergency diagnosis and treatment of adult meningitis. Lancet Infect Dis 7(3):191-200

33. Ziai WC, Lewin JJ, 3rd. Update in the diagnosis and management of central nervous system infections. Neurol Clin 2008;26(2):42768, viii.

34. Grijseels EW, Bouten MJ, Lenderink T, Deckers JW, Hoes AW, Hartman JA et al (1995) Pre-hospital thrombolytic therapy with either alteplase or streptokinase. Practical applications, complications and long-term results in 529 patients. Eur Heart J 16 (12): $1833-1838$

35. Boersma E, Maas AC, Deckers JW, Simoons ML (1996) Early thrombolytic treatment in acute myocardial infarction: reappraisal of the golden hour. Lancet 348(9030):771-775

36. Newby LK, Storrow AB, Gibler WB, Garvey JL, Tucker JF, Kaplan AL et al (2001) Bedside multimarker testing for risk stratification in chest pain units: The chest pain evaluation by creatine kinase-MB, myoglobin, and troponin I (CHECKMATE) study. Circulation 103(14):1832-1837

37. Collins SP, Levy PD, Lindsell CJ, Pang PS, Storrow AB, Miller $\mathrm{CD}$ et al (2009) The rationale for an acute heart failure syndromes clinical trials network. J Card Fail 15(6):467-474

38. Rivers E, Nguyen B, Havstad S, Ressler J, Muzzin A, Knoblich B et al (2001) Early goal-directed therapy in the treatment of severe sepsis and septic shock. N Engl J Med 345 (19):1368-1377

39. Pope JV, Jones AE, Gaieski DF, Arnold RC, Trzeciak S, Shapiro NI (2009) Multicenter Study of Central Venous Oxygen Saturation $(\mathrm{ScvO}(2))$ as a Predictor of Mortality in Patients With Sepsis. Ann Emerg Med

40. Busch M (2006) Portable ultrasound in pre-hospital emergencies: a feasibility study. Acta Anaesthesiol Scand 50(6):754-758

41. Kimberly HH, Shah S, Marill K, Noble V (2008) Correlation of optic nerve sheath diameter with direct measurement of intracranial pressure. Acad Emerg Med 15(2):201-204

42. Le A, Hoehn ME, Smith ME, Spentzas T, Schlappy D, Pershad J (2009) Bedside sonographic measurement of optic nerve sheath diameter as a predictor of increased intracranial pressure in children. Ann Emerg Med 53(6):785-791

43. Moretti R, Pizzi B, Cassini F, Vivaldi N (2009) Reliability of optic nerve ultrasound for the evaluation of patients with spontaneous intracranial hemorrhage. Neurocrit Care 11(3):406-410

44. Kost GJ, Korte B, Beyette FR Jr, Gaydos C, Weigl B (2008) The NIBIB Point of Care Technologies Research Network Center Themes and Opportunities for Exploratory POC Projects. Point of Care: The Journal of Near-Patient Testing and Technology 7(1):41 